\title{
Genetic dynamics in the sand fly (Diptera: Psychodidae) nuclear and mitochondrial genotypes: evidence for vector adaptation at the border of Iran with Iraq
}

Sahar Ebrahimi ${ }^{1,2}$, Ali Bordbar $^{1,2}$ and Parviz Parvizi ${ }^{1,2^{*}}$

\begin{abstract}
Background: Our investigation uses nucleotide variations of the genera Phlebotomus and Sergentomyia using the EF-1a and Cyt b genotype regions to describe the sand fly fauna and genetic aspects collected at war-torn sites of the Khuzestan boundary between Iraq and Iran.

Methods: All sand fly species were characterized using molecular genetics. The field work was conducted in six districts including 24 locations in remote areas for three years at the peak of sand fly activity during cutaneous leishmaniasis (CL) transmission seasons. The distribution of $\mathrm{CL}$ vectors was determined based on the climatic regionalization using the kriging method in ArcGIS model. DNA of sand fly pools were screened via polymerase chain reaction ( $P C R$ ) using neutrality (Tajima's $D$ ) and neutral allele frequency (Fu's $F_{s}$ ) tests to measure the effect of randomly evolving DNA sequence on the genetic diversity of sand fly populations in response to habitat fragmentation and landscape modification.

Results: Among the 1213 specimens, ten species were identified based on morphology. The non-native species Phlebotomus sergenti was unequivocally found for the first time in the studied regions. Nucleotide substitutions of sand fly sequences varied most in the most disrupted districts (Dashte-Azadegan and Abadan; disparity index test: $P<0.05)$. The haplotypes of Cyt $b$ from the subgenus Sergentomyia and $P$. papatasi revealed more heterogeneity (Tajima's $D>+2$ ) than $P$. alexandri $(D>+1)$, which suggests widespread heteroplasmic mitochondrial DNA mutations in the same mtDNA gene among different sand fly species. Subgenus Sintonius exhibited greater fitness $(D=0)$ and (neutrality test; $P>0.05$ ) no evidence of selection. The sequence of the nuclear gene EF-1a indicated similar nucleotide differences, as observed for the Cyt $b$ gene, in all sand fly species, but lower levels of polymorphisms $(D>+1)$ were observed compared with the mitochondrial Cyt $b$ gene $(D>+2)$ in the subgenus Sergentomyia.

Conclusion: Our findings describe random nucleotide diversity in the Phlebotomus and Sergentomyia population gene pools due to recent anthropogenic influence. A phylogenetic analysis showed that the closely related species are positioned in monophyletic clades, except for the subgenus Sergentomyia and P. sergenti, and highlights the importance of haplotype variations for the development of adaptability.
\end{abstract}

Keywords: Cyt b gene, EF-1a gene, Genetic adaptation, Iran-Iraq boundaries, Landscape fragmentation, Molecular analyses, Phlebotominae

\footnotetext{
* Correspondence: parpparvizi@yahoo.com

${ }^{1}$ Molecular Systematics Laboratory, Parasitology Department, Pasteur Institute

of Iran, Tehran, Iran

${ }^{2}$ Microbiology Research Center (MRC), Pasteur Institute of Iran, Tehran, Iran
} 


\section{Background}

Hematophagous phlebotomine sand fly females are medically important but only some are proven leishmaniasis vectors with critical vectorial capacity and competency [1]. Sand flies transmit Leishmania spp. through their geographical distribution due to landscape fragmentation, migration, environmental and climatic oscillations. Certain fundamental criteria support the notion that sand flies are potential vectors: the capacity of sand flies to use their midgut receptor to attach a specific Leishmania species, behavioral or genetic diversification, vector-parasite coevolution and the adaptive manipulation of their interactions [2-5].

In a noteworthy Zoonotic Cutaneous Leishmaniasis (ZCL) focus, in a tropical climate region in southwestern Iran, Khuzestan Province has suffered from the Iraq-Iran war (from 22 September 1980 to 20 August 1988) and has recently been involved in Daesh terrorist destruction, which has led to ecological instability in the region, providing adequate conditions for flourishing heterogeneity and allowing for great diversity among sand fly species. The ZCL incidence was low during war time, but it suddenly soared among soldiers with over 10,000 annual cases reported from the public health centers of Khuzestan [6]. The prevalence range 1.8-37.9\% and incidence rate of $69,000-113,300 \mathrm{CL}$ cases were reported by the Iran Ministry of Health and Medical Education and as WHO estimates, respectively [7, 8]. Despite the large and diverse sand fly distribution range in the geographical borders of the Khuzestan Province between Iran and Iraq, the sand fly species and the population structure of CL sand flies are not well-known. The commemoration of a religious man, Imam Hussein, is one of the largest religious gatherings in the world and a large number of Iranian and/or Afghan people (about two million pilgrims annually) walk through CL areas from across the Iranian boundaries (Khuzestan and Ilam Provinces) to Iraq. Indigenous and traveler populations are at risk for Leishmania infection by different sand fly species in many active transmission areas between expatriates and visitors. Simultaneous utilization of the sequence of applying both mitochondrial Cytochrome $b(C y t b)$ and conserved nuclear marker elongation factor-1 $\alpha(E F-1 \alpha)$ as proper DNA markers have already provided data for analyzing the phylogenetic relationship among sand fly species [9]. These genes provide sufficient information to discern the species' ancestor population in the natural habitat of phlebotomine sand flies [10].

The maternally-inherited mitochondrial $C y t b$ gene, reflects the evolutionary history because of high evolution rate, lack of recombination and clonal inheritance [11, 12]. The $C y t b$ gene has also been used for analyzing the genetic divergence within and among phlebotomine sand fly populations, mainly due to its high variability $[1,13,14]$.
The nuclear gene, $E F-1 \alpha$, has a remarkable character to detect heterozygosity, haplotype diversity and genetic variation. The $E F-1 \alpha$ gene has been identified as a potentially useful gene for studies of high-level phylogenetic relationships, particularly in insects $[10,15]$. Many reports consider $E F-1 \alpha$ as a proper marker for methodological and analytical challenges in systematic biology and molecular phylogeny [16-18].

In this study, we investigate species of the genus Phlebotomus as the main vectors of leishmaniasis as well as species of the genus Sergentomyia in studied areas. It is noticeable that DNA of $L$. major and L. tropica was definitely identified from Sergentomyia spp. by PCR-RFLP and ITS1 gene sequencing in Ghana and West Africa [19]. Initial morphological identification of sand fly species followed by molecular confirmation allowed us to improve effective, targeted and control measurements. To our knowledge, although some combined data from the markers of mitochondrial genes (e.g. Cyt b, Cytochrome $c$ oxidase subunit $I$ and $N A D H 4)$ and/or nuclear genes (e.g. $E F-1 \alpha$ and ribosomal DNA) $[10,12,20]$ have achieved accurate information for the sand fly identification and population genetics, the neutrality test of loci has rarely been investigated [21]. Whereas sand flies pose substantial human health concerns, the objectives of this study were to identify collected sand flies to the species level using morphological and molecular characteristics and to determine the sand flies' evolutionary relationships with landscape fragmentation using neutrality and phylogenetic analyses along the $c .420 \mathrm{~km}$ Khuzestan borders.

\section{Methods}

\section{Origin, collection and morphological identification}

Sand fly species were caught on a large geographical scale in Khuzestan Province, which is located southwest of Iran $\left(30^{\circ} 20^{\prime} 21^{\prime \prime} \mathrm{N}\right.$ to $31^{\circ} 33^{\prime} 29^{\prime \prime} \mathrm{N}$ and $48^{\circ} 18^{\prime} 15^{\prime \prime} \mathrm{E}$ to $\left.48^{\circ} 10^{\prime} 51^{\prime \prime} \mathrm{E}\right)$, bordering with Basrah and Maysan, Iraq provinces in the west and the Persian Gulf in the south (Fig. 1). The climate changes and type of weather in the studied areas differ from the other CL foci conditioned in Iran, with humidity ranging from 50 to $85 \%$, temperature ranging from 20 to $60{ }^{\circ} \mathrm{C}$ and a monthly average precipitation between 17 to $25 \mathrm{~mm}$. Sand fly specimens were collected from 24 sites adjacent to Iraq's borders in western parts of Khuzestan within approximately $10,406 \mathrm{~km}^{2}$ during their seasonal activity in late May to late November 2011-2014 (Fig. 1). The deterministic spatial interpolation was performed based on ArcGIS model using kriging method and bioclimatic variables obtained from Ebrahimi et al. data [22]. The climatic regionalization was used with the principal component analysis (PCA) and the clustering integration method (CIM) to obtain the appropriate classification.

Sticky sheets of paper and CDC miniature light traps were placed $1-1.5 \mathrm{~m}$ above the ground to sample the 


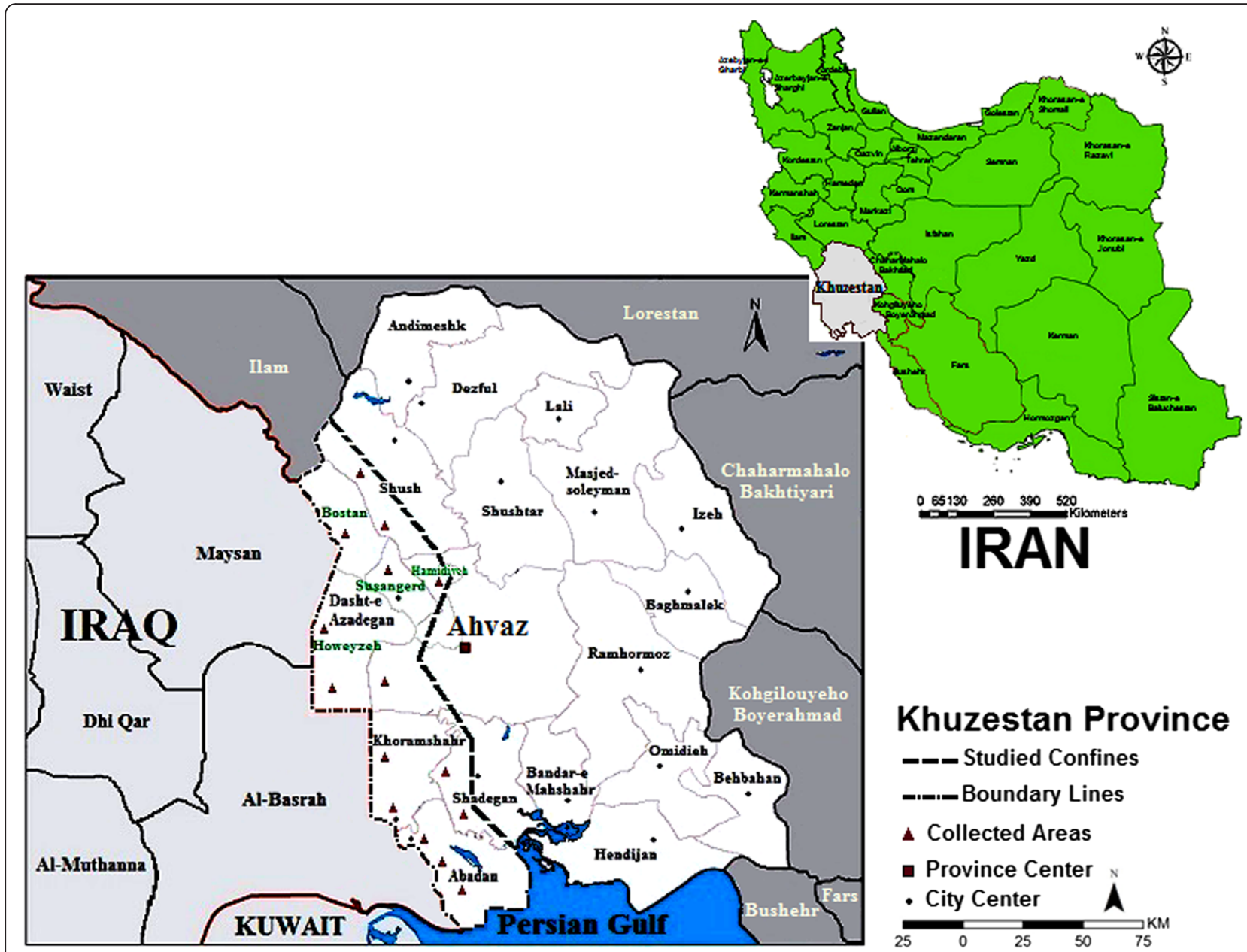

Fig. 1 Map of Khuzestan Province of Iran with the collection sites for the sand flies caught across the boundary lines with Iraq (the main districts are confined within the dashed outline)

sand flies and were deployed overnight (before dusk until dawn). Sand fly traps were placed in a natural field of destroyed war remnants, natural and artificial holes and crevices, agricultural fields, gerbil burrow entrances, new developing urban areas, rural areas adjacent to the boundary lines, domestic animal shelters and around houses close to gerbil burrows two times per month (5 days each time) $[23,24]$. All sand flies were examined and identified based on morphological characteristics of the head and abdominal terminalia using compound microscopy $(400 \times)$. The genitalia of each sand fly were carefully removed using micro-needles and slides-mounted in Berlese's fluid following dissection using sterilized forceps [24].

\section{DNA extraction and PCR amplification of the $C y t b$ and EF-1a gene regions}

Because a certain level of ambiguity and/or similarity appeared among female sand fly morphological characteristics of closely related species; the collected sand flies were molecularly characterized using the $C y t b$ and $E F-1 \alpha$ gene regions. PCR fragments were amplified as previously described $[10,11,14,25]$. The total DNA of the dissected thorax and anterior abdomen of individual sand flies was extracted using the modified method of Ish-Horowicz [26], GeNet Bio and a DynaBio ${ }^{\text {tm }}$ Kit (Bioneer corporation, Seoul, Korea and Takapouzist corporation, Tehran, Iran). One hundred nano grams of each purified DNA sample was cycle-sequenced using an AccuPower ${ }^{\circ}$ DNA Sequencing Kit from Bioneer and 3730xl/Bioneer 3730xl sequencing systems with $3.2 \mathrm{pmol}$ of the same primers used for PCR. Nucleotide sequence data and new identified haplotypes of Phlebotomus and Sergentomyia spp. reported in this paper are submitted to the GenBank, EMBL, and DDBJ databases under accession numbers KX024717-KX024728; KX06 7785-KX067788 and KX088453-KX088456 (Cyt $b$ gene) and KX099722-KX099736 (EF-1 $\alpha$ gene).

Molecular genetics and phylogenetic data analyses After sequencing the PCR amplicons (both strands), all DNA sequences were exported to Sequencher ${ }^{\text {tax }}$ 
v.4.1.4 for PC (Gene Codes Corporation) to identify the limits of the open reading frames (ORFs). The primers were trimmed from the sequences, aligned based on deduced amino acid sequences and visually edited, which yielded sequences between 758-790 nucleotides in length for the Cyt $b$ gene region and 430497 for the $E F-1 \alpha$ including the primers. Visual analyses were used to assess heterogeneity and/or single nucleotide polymorphisms (SNPs) in both directions. A disparity index test for substitution pattern homogeneity was calculated with 500 Monte Carlo replications within $P$. papatasi, $P$. alexandri and Sergentomyia spp. populations $(P<0.05)[27]$.

The phylogenies were reconstructed to evaluate the species status and establish the relationships from the common and new haplotypes of the EF-1 $\alpha$ and $C y t b$ Phlebotominae sand fly sequences. A maximum likelihood (ML) analysis using a heuristic search through stepwise addition of 100 random replicates and bootstrapping with 1000 replicates were performed using the MEGA 5.05 package based on the Tamura-Nei model of a nucleotide substitution search from Clustal W [28]. To group the various sand fly species, the phylogenetic maximum likelihood (PhyML) [29] program was run to obtain a maximum likelihood tree, and nonparametric ML bootstrap was used with 1000 replicates.

To test the differences in sand fly species abundance, statistical analyses were performed on all of the Phlebotomus species individually as main vectors susceptible to maintaining the transmission cycle of Leishmania parasites in the region and Sergentomyia spp., to be nonborne disease vectors. The MEGA 5.05 program was also used to calculate the number of variable nucleotide sites, nucleotide diversity (average number of nucleotide differences per site between any two randomly selected sequences) and the transition/transversion ratio $(R)$. The potential selection pressure of the protein-coding sequences was determined using the $\mathrm{dN} / \mathrm{dS}$ ratio test and a Z-test for selection based on the Nei-Gojobori method. DnaSp 5.10.01 was used to evaluate haplotype diversity (HD) and to analyze the value of Fu's $F_{\mathrm{s}}$ (neutrality and allele frequency) for each species [30].

\section{Results}

\section{Sand fly collection, morphological identification and} sequenced specimens

For this investigation, we identified ten species among Phlebotomus and Sergentomyia sand flies caught in 23 villages and a riverside next to the Khuzestan boundaries (Table 1). Three species of the genus Phlebotomus ( $P$. papatasi, $P$. alexandri and $P$. sergenti) and seven species of the genus Sergentomyia (S. sintoni, S. antennata, S. dentata, S. baghdadis, S. iranica, S. clydei and S. tiberiadis) were found among a total of 1213 sand flies, including 893 Phlebotomus and 320 Sergentomyia specimens (Table 1). The studied area was identified as the low rainfall and high relative humidity zone on the basis of the effects of principal factors on climatic variables obtained from Ebrahimi et al. [22] (Fig. 2). We also successfully collected $P$. sergenti in the Dashte-Azadegan district and Abadan for the first time (Table 1).

Of 145 sequenced specimens for the Cyt $b$ (78) and EF$1 \alpha$ (67) gene regions, 108 sequences could be analyzed for Phlebotomus spp. $(n=59)$ including P. papatasi $(n=16)$ and $P$. alexandri $(n=15)$ for Cyt $b ; P$. papatasi $(n=15)$ and P. alexandri $(n=11)$ for $E F-1 \alpha$ and two sequences of $P$. sergenti $(n=2)$ for both gene regions; and a total of 49 sequences from Sergentomyia specimens including $S$. antennata $(n=2)$ and $(n=3)$ for $S$. dentata, S. tiberiadis, $S$. clydei, S. iranica and S. baghdadis for both Cyt $b$ and EF-1a gene regions, and S. sintoni ( $n=11$ and $n=10)$ for the $C y t$ $b$ and EF-1 $\alpha$ markers, respectively (Table 1$)$. Altogether, 37 sequenced specimens of $P$. papatasi $(n=20)$ and $P$. alexandri $(n=17)$ did not have enough DNA or were not interpretable after resequencing.

\section{Molecular and statistical analyses of the Cyt $b$ gene region}

Our trimmed sequences, along with those deposited in GenBank, were analyzed to compare and identify new haplotypes (Tables 2, 3). After aligning and pruning all of the sequences, the total lengths of the Phlebotomus and Sergentomyia species were contained within 632790 base pairs. The species $P$. sergenti and S. iranica were not statistically analyzed due to the limited number of sand flies caught in the studied areas (Table 4). Further, a single species, S. baghdadis (KH300), which does not transmit disease, was not statistically analyzed. The sequenced region of the $C y t b$ gene was $\mathrm{A}-\mathrm{T}$ rich in all species (Table 4).

Phlebotomus papatasi had four unique haplotypes (KH417, KH419, KH421 and KH486) and a common haplotype of $\mathrm{KH} 382, \mathrm{KH} 535$ and other remaining sequences that had a $100 \%$ similarity compared to haplotypes from Italy (GenBank accession nos. HM992926 and HM992927) (Table 2). Edited P. alexandri sequences revealed five unique haplotypes in two similar groups isolated from Abadan (KH384 and KH528) and from Dashte-Azadegan (KH407, KH408 and KH410) (Table 2). The haplotype diversity (HD) for genus Sergentomyia was $P=0.987$ and three species of the subgenus Sergentomyia were characterized molecularly which showed four, two and one unique haplotype/s for S. sintoni, S. antennata and $S$. dentata, respectively (Table 2). In the subgenus Sintonius, three haplotypes were observed for the two species, $S$. tiberiadis and $S$. clydei (Table 2). Additionally, two new haplotypes were obtained for S. iranica (KH519 and KH135), with three 
Table 1 Fauna and distribution of sandflies collected from six districts along the Khuzestan boundaries, characterized based on morphological feature examinations and molecular analyses (M: male, F: female; data on sandflies that were not collected are not shown in this table)

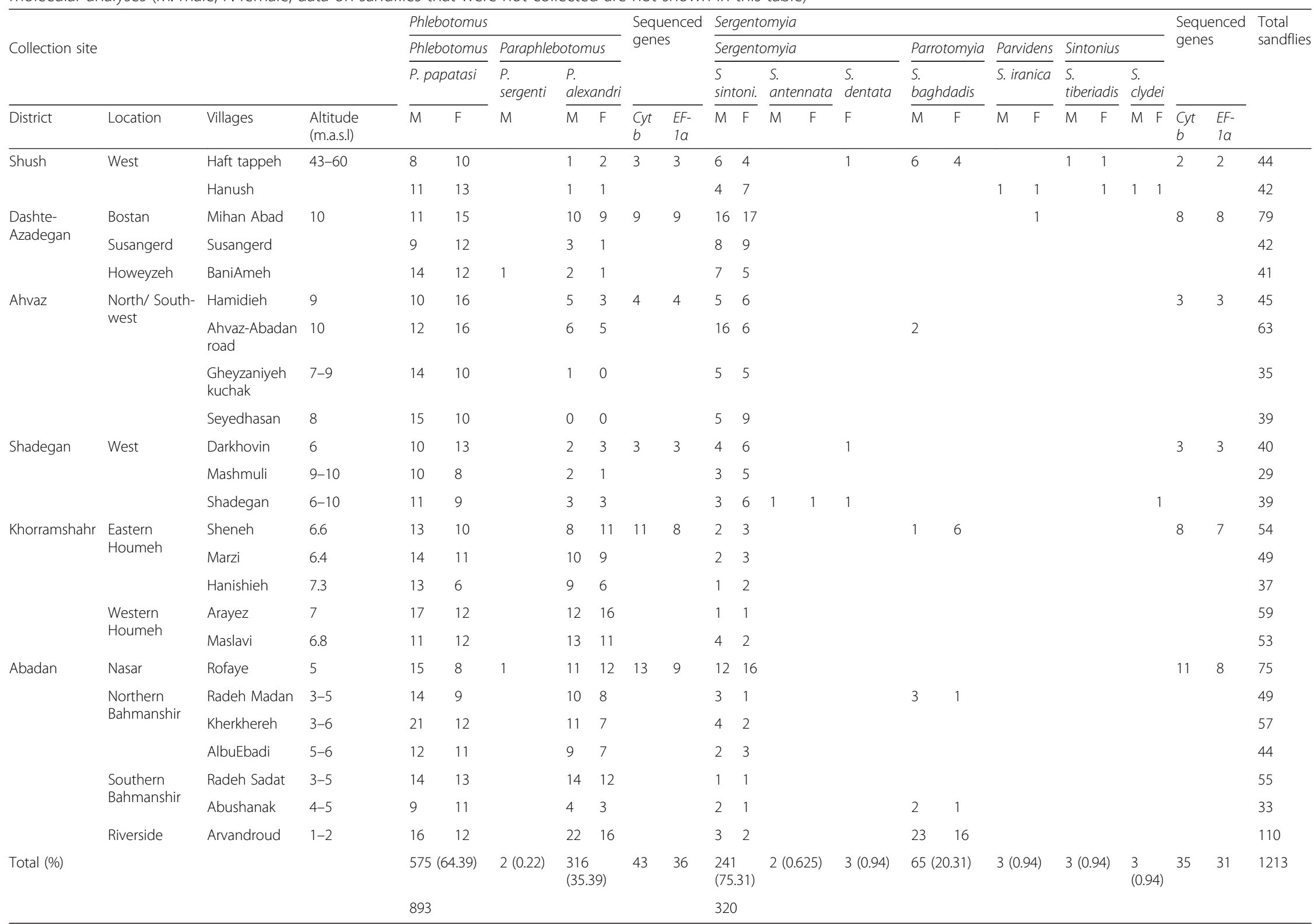




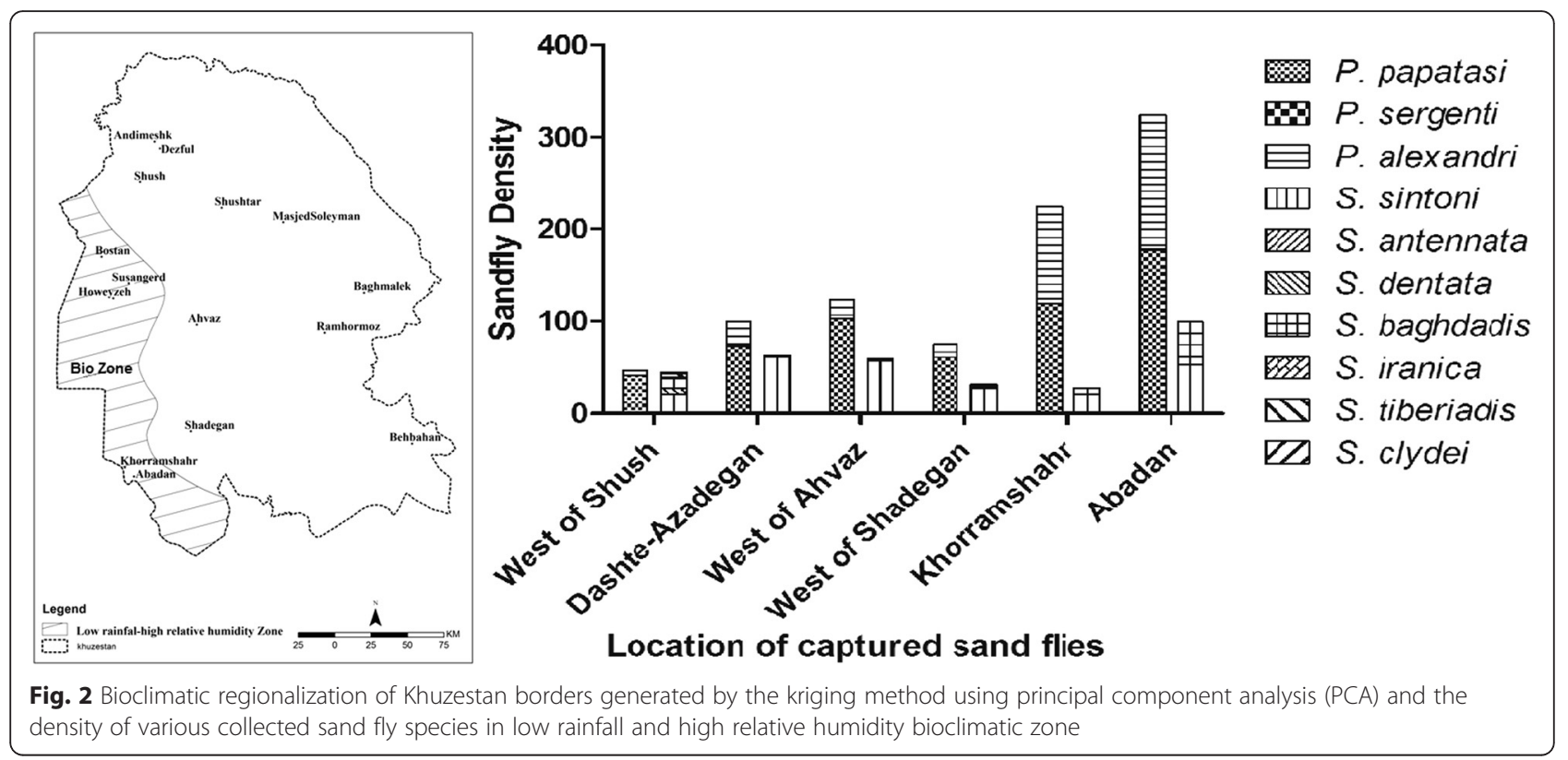

A-T transversions and 18 transitions in the sequences (Table 2). The nucleotide diversity within the haplotypes of $P$. papatasi in Khorramshahr and Abadan and the subgenus Sergentomyia in all villages was generally significant (Tajima's $D>+2$ ). The results of the neutrality test for $P$. alexandri haplotypes were considerable $(D>$ $+1, P=1.876884$ ) but lower than that for $P$. papatasi $(D>+2, P=2.391599)$, which indicates that the $P$. alexandri population is attempting to undergo equilibrium among gene frequencies and balancing selection (Table 4). The percentage of similarities and divergences among populations of Phlebotomus and Sergentomyia are indicated in Fig. 3.

A statistical analysis of the selection pressures between the $P$. papatasi sequences clearly shows that the $\mathrm{dN} / \mathrm{dS}$ ratios were strongly biased toward synonymous mutations (the $\mathrm{dN} / \mathrm{dS}$ ranged from $P=0.008$ of $\mathrm{KH} 486$ / KH419 to $P=0.346$ of KH486/KH417) in a total of 215 positions in the final dataset. This phenomenon is likely due to counterselection of deleterious mutations during the $C y t b$ gene evolution. Additionally, the neutrality test results were not considerable $(P>0.05)$ between the Sintonius spp. sequences.

\section{Molecular and statistical analyses of the EF-1a gene region}

The total length of the $E F-1 \alpha$ gene region was 497 bp for all species. The $E F-1 \alpha$ gene exhibits higher GC content than the Cyt $b$ gene in the subgenus Sergentomyia (Table 4). The haplotypes identified from the $E F-1 \alpha$ gene for each sand fly species take the following order: $P$. papatasi, P. alexandri and S. tiberiadis (two), and S. sintoni (four) haplotypes. Additionally, S. antennata, S. dentata, S. baghdadis and S. clydei each featured a unique sequence. Moreover, the KH421 sequence from $P$. papatasi exhibited 13 nucleotide differences compared with a previously detected sequence from the villages of Isfahan and Golestan areas (less affected regions by war), Iran (IRN377, GenBank accession no. EF416843), and S. sintoni haplotypes KH (133, 134, 322 and 327) exhibited 15 differences compared with the Iranian haplotype (IRN341, GenBank accession no. EF416846). Sequences for S. iranica (KH519 and KH135) were not interpretable after resequencing.

Homogeneity was examined based on whether differences in base composition bias between sequences within the subgenus Sergentomyia (Disparity index test) were significant $(P<0.05)$; significant differences were only observed between the KH108 (S. antennata) and KH134 (S. sintoni) sequences $(P=0.016)$. The remaining sequence estimates were equal $(P=1.000)$. The Z-test for selection pressure using the variance bootstrap method $(\mathrm{dN} / \mathrm{dS})$ was remarkable $(P<0.05)$ for closely related species of $\mathrm{KH} 108 / \mathrm{KH} 133$, KH322 $(P=0.046, S$. antennata and $S$. sintoni $) ; \mathrm{KH} 108 / \mathrm{KH} 327$ $(P=0.020$, S. antennata and $S$. sintoni $)$ and KH327/KH134 $(P=0.026, S$. sintoni). However, this analytical test was only significant between KH493 and KH108 (S. dentata and S. antennata) for the $C y t b$ mitochondrial gene within the subgenus Sergentomyia.

The phylogenetic tree of approximately most caught sand flies shows similarity among different haplotypes in the related clade; however, the subgenus Sergentomyia was not resolved as a monophyletic clade, which is consistent with our previous findings (Figs. 4, 5) [10]. The statistical correlations were not significant 
Table 2 All variant haplotypes of the Cyt b gene and indicated SNPs in sandflies showing inheritance of one or other parental allele isolated from Iran-Iraq boundaries (Unamplified sequences and identities in relevant positions are denoted by dashes and dots, respectively; M: male; F: female)

\begin{tabular}{|c|c|c|c|c|c|c|c|c|c|c|c|c|c|c|c|c|c|c|c|c|c|c|c|c|}
\hline \multirow[t]{4}{*}{ Species } & \multirow[t]{4}{*}{ Origin } & \multirow[t]{4}{*}{ Sex } & \multirow[t]{4}{*}{ Haplotype (GenBank acc. no.) } & \multicolumn{21}{|c|}{ Position of nucleotide substitution } \\
\hline & & & & 1 & 1 & 3 & 5 & 7 & 7 & 7 & & & & & & & & & & & & & & \\
\hline & & & & 7 & 9 & 4 & 6 & 3 & 4 & 4 & & & & & & & & & & & & & & \\
\hline & & & & 8 & 8 & 2 & 7 & 2 & 3 & 4 & & & & & & & & & & & & & & \\
\hline \multirow[t]{5}{*}{ P. papatasi } & Abadan (Italy) & M & KH382/535 (HM992927/HM992926) & G & $\mathrm{T}$ & $\mathrm{C}$ & A & $C$ & A & $\mathrm{T}$ & & & & & & & & & & & & & & \\
\hline & Abadan & $\mathrm{F}$ & KH417 (KX024717) & A & . & . & . & . & . & . & & & & & & & & & & & & & & \\
\hline & Khorramshahr & $\mathrm{F}$ & KH419 (KX024719) & G & C & . & G & $\mathrm{T}$ & C & & & & & & & & & & & & & & & \\
\hline & Abadan & M & KH421 (KX024718) & . & $\mathrm{T}$ & . & A & C & A & C & & & & & & & & & & & & & & \\
\hline & & M & KH486 (KX024720) & & & $\mathrm{T}$ & G & & & $\mathrm{T}$ & & & & & & & & & & & & & & \\
\hline \multirow[t]{12}{*}{ P. alexandri } & $\mathrm{O}$ & S & $\mathrm{H}$ & & & & & 2 & 2 & 3 & 5 & 5 & 5 & 6 & 6 & 6 & & & & & & & & \\
\hline & & & & 3 & 4 & 5 & 8 & 0 & 6 & 0 & 0 & 5 & 5 & 2 & 4 & 7 & & & & & & & & \\
\hline & & & & 9 & 5 & 4 & 2 & 8 & 3 & 4 & 8 & 4 & 9 & 8 & 6 & 9 & & & & & & & & \\
\hline & Abadan & $\mathrm{F}$ & KH384 (KX024721) & A & $\mathrm{T}$ & $\mathrm{T}$ & G & $\mathrm{T}$ & A & A & T & $\mathrm{T}$ & G & A & $\mathrm{T}$ & C & & & & & & & & \\
\hline & & M & KH528 (KX024725) & $\mathrm{T}$ & A & $C$ & A & $C$ & G & G & A & $C$ & A & G & A & $\mathrm{T}$ & & & & & & & & \\
\hline & $\mathrm{O}$ & S & $\mathrm{H}$ & & 2 & 2 & 4 & 5 & 7 & 7 & 7 & 7 & 7 & 7 & 7 & & & & & & & & & \\
\hline & & & & 4 & 1 & 2 & 1 & 2 & 0 & 2 & 2 & 4 & 4 & 5 & 6 & & & & & & & & & \\
\hline & & & & 5 & 1 & 3 & 2 & 7 & 9 & 2 & 4 & 4 & 5 & 4 & 0 & & & & & & & & & \\
\hline & Germany & & (HM803186) & - & - & - & - & A & T & $\mathrm{T}$ & A & A & T & G & G & & & & & & & & & \\
\hline & Bostan & M & KH407 (KX024722) & T & $\mathrm{T}$ & $\mathrm{T}$ & $\mathrm{T}$ & $C$ & & & & . & & & & & & & & & & & & \\
\hline & & F & KH410 (KX024724) & A & . & . & & . & . & . & . & . & $C$ & A & A & & & & & & & & & \\
\hline & Howeyzeh & M & KH408 (KX024723) & & G & $C$ & $C$ & A & $C$ & C & $\mathrm{T}$ & $C$ & & & . & & & & & & & & & \\
\hline \multirow[t]{7}{*}{ S. sintoni } & $\mathrm{O}$ & S & $\mathrm{H}$ & & 2 & 2 & 3 & 6 & & & & & & & & & & & & & & & & \\
\hline & & & & 3 & 7 & 8 & 0 & 0 & & & & & & & & & & & & & & & & \\
\hline & & & & 3 & 9 & 0 & 7 & 1 & & & & & & & & & & & & & & & & \\
\hline & Shadegan & M & KH133 (KX067785) & G & G & G & $\mathrm{T}$ & $\mathrm{T}$ & & & & & & & & & & & & & & & & \\
\hline & & $\mathrm{F}$ & KH134 (KX067786) & A & . & . & . & . & & & & & & & & & & & & & & & & \\
\hline & Bostan & M & KH322 (KX067787) & . & A & A & $C$ & C & & & & & & & & & & & & & & & & \\
\hline & & $\mathrm{F}$ & KH327 (KX067788) & . & . & . & $\mathrm{T}$ & $\mathrm{T}$ & & & & & & & & & & & & & & & & \\
\hline \multirow[t]{5}{*}{ S. antennata } & $\mathrm{O}$ & S & $\mathrm{H}$ & & & 2 & 2 & 4 & 6 & 6 & 6 & 6 & & & & & & & & & & & & \\
\hline & & & & 5 & 8 & 2 & 3 & 7 & 3 & 7 & 7 & 8 & & & & & & & & & & & & \\
\hline & & & & 4 & 4 & 2 & 1 & 7 & 0 & 0 & 5 & 0 & & & & & & & & & & & & \\
\hline & Shadegan & M & KH108 (KX099719) & A & $\mathrm{T}$ & G & C & C & T & $\mathrm{T}$ & $C$ & T & & & & & & & & & & & & \\
\hline & & $\mathrm{F}$ & KH154 (KX099720) & T & C & A & $\mathrm{T}$ & $\mathrm{T}$ & $C$ & C & $\mathrm{T}$ & A & & & & & & & & & & & & \\
\hline \multirow[t]{5}{*}{ S. iranica } & $\mathrm{O}$ & S & $\mathrm{H}$ & & & & & 1 & 1 & 2 & 2 & 3 & 3 & 3 & 3 & 3 & 3 & 4 & 4 & 5 & 5 & 5 & 5 & 6 \\
\hline & & & & 4 & 7 & 7 & 8 & 0 & 1 & 5 & 7 & 0 & 2 & 3 & 3 & 5 & 8 & 1 & 2 & 1 & 1 & 7 & 9 & 2 \\
\hline & & & & 2 & 2 & 6 & 8 & 8 & 1 & 2 & 3 & 1 & 7 & 0 & 1 & 8 & 1 & 7 & 6 & 0 & 9 & 9 & 7 & 7 \\
\hline & Susangerd & $\mathrm{F}$ & KH135 (KX024727) & G & C & $C$ & $\mathrm{~T}$ & A & A & $\mathrm{T}$ & T & G & $A$ & $A$ & $\mathrm{~T}$ & C & C & G & G & T & C & C & G & C \\
\hline & Bostan & M & KH519 (KX024728) & A & $\mathrm{T}$ & $\mathrm{T}$ & $C$ & G & $\mathrm{T}$ & C & $C$ & A & $\mathrm{T}$ & $\mathrm{T}$ & C & $\mathrm{T}$ & $\mathrm{T}$ & A & A & $C$ & $\mathrm{~T}$ & $\mathrm{~T}$ & A & $\mathrm{T}$ \\
\hline \multirow[t]{5}{*}{ S. tiberiadis } & $\mathrm{O}$ & S & $\mathrm{H}$ & 1 & 1 & 3 & 6 & & & & & & & & & & & & & & & & & \\
\hline & & & & 0 & 5 & 6 & 9 & & & & & & & & & & & & & & & & & \\
\hline & & & & 8 & 0 & 1 & 4 & & & & & & & & & & & & & & & & & \\
\hline & Haft tappeh & $\mathrm{F}$ & KH305 (KX088454) & G & C & $\mathrm{T}$ & $\mathrm{T}$ & & & & & & & & & & & & & & & & & \\
\hline & Hanush & $\mathrm{F}$ & KH307 (KX088455) & A & $T$ & C & C & & & & & & & & & & & & & & & & & \\
\hline
\end{tabular}


Table 3 Variable haplotypes found in the alignment of species of Phlebotomus and Sergentomyia based on molecular analyses of the EF-1a nuclear gene obtained from Iran-Iraq boundaries

\begin{tabular}{|c|c|c|c|c|c|c|c|c|c|c|c|c|c|c|c|c|c|c|c|c|c|c|c|}
\hline \multirow{2}{*}{$\begin{array}{l}\text { Gene } \\
\text { EF-1a }\end{array}$} & \multirow{2}{*}{$\begin{array}{l}\text { Species } \\
\text { P. papatasi }\end{array}$} & \multirow[t]{2}{*}{ Origin } & \multirow[t]{2}{*}{ Haplotype (GenBank acc. no.) } & \multicolumn{20}{|c|}{ Position of nucleotide substitution } \\
\hline & & & & & & 1 & 1 & 2 & 2 & 2 & 3 & 3 & 3 & 3 & 4 & 4 & 4 & 4 & 4 & 4 & 4 & 4 & 4 \\
\hline & & & & 3 & 7 & 0 & 6 & 0 & 2 & 7 & 3 & 8 & 8 & 9 & 0 & 0 & 1 & 1 & 2 & 2 & 2 & 3 & 3 \\
\hline & & & & 9 & 8 & 8 & 2 & 4 & 2 & 0 & 9 & 6 & 7 & 5 & 7 & 9 & 5 & 9 & 0 & 2 & 4 & 0 & 2 \\
\hline & & Abadan & KH382 (KX099735) & $\mathrm{T}$ & C & C & A & $A$ & T & $\mathrm{T}$ & C & $\mathrm{T}$ & $C$ & $C$ & $A$ & G & $C$ & A & G & $\mathrm{T}$ & $\mathrm{T}$ & A & G \\
\hline & & & KH421 (KX099736) & G & $\mathrm{T}$ & $A$ & G & G & C & $C$ & $\mathrm{~T}$ & G & $A$ & A & $\mathrm{T}$ & A & G & $\mathrm{T}$ & $C$ & $A$ & G & T & A \\
\hline & P. alexandri & $\mathrm{O}$ & $\mathrm{H}$ & 2 & 2 & 2 & 2 & 3 & 3 & 3 & 3 & & & & & & & & & & & & \\
\hline & & & & 4 & 4 & 9 & 9 & 0 & 1 & 5 & 8 & & & & & & & & & & & & \\
\hline & & & & 3 & 9 & 1 & 7 & 6 & 8 & 5 & 1 & & & & & & & & & & & & \\
\hline & & Abadan & KH384 (KX099733) & A & G & $\mathrm{T}$ & G & $\mathrm{T}$ & $\mathrm{T}$ & C & A & & & & & & & & & & & & \\
\hline & & Howeyzeh & KH408 (KX099734) & G & A & $A$ & C & $C$ & $A$ & $\mathrm{~T}$ & G & & & & & & & & & & & & \\
\hline & S. sintoni & O & $\mathrm{H}$ & & & 2 & 4 & 4 & 4 & 4 & 4 & & & & & & & & & & & & \\
\hline & & & & 3 & 4 & 9 & 4 & 5 & 6 & 6 & 7 & & & & & & & & & & & & \\
\hline & & & & 6 & 2 & 7 & 7 & 9 & 2 & 8 & 1 & & & & & & & & & & & & \\
\hline & & Shadegan & KH133 (KX099722) & C & A & $\mathrm{T}$ & $\mathrm{T}$ & C & G & $\mathrm{T}$ & $A$ & & & & & & & & & & & & \\
\hline & & Bostan & KH322 (KX099723) & & . & . & $C$ & . & . & . & & & & & & & & & & & & & \\
\hline & & Shadegan & KH134 (KX099724) & G & C & G & . & $A$ & C & G & C & & & & & & & & & & & & \\
\hline & & Bostan & KH327 (KX099725) & & A & . & . & . & . & . & & & & & & & & & & & & & \\
\hline & S. tiberiadis & $\mathrm{O}$ & $\mathrm{H}$ & & 2 & 3 & 3 & & & & & & & & & & & & & & & & \\
\hline & & & & 5 & 9 & 1 & 9 & & & & & & & & & & & & & & & & \\
\hline & & & & 7 & 4 & 5 & 0 & & & & & & & & & & & & & & & & \\
\hline & & Haft tappeh & KH305 (KX099726) & T & C & $C$ & G & & & & & & & & & & & & & & & & \\
\hline & & Hanush & KH307 (KX099727) & C & $\mathrm{T}$ & $\mathrm{T}$ & $A$ & & & & & & & & & & & & & & & & \\
\hline
\end{tabular}

Table 4 Tajima's D neutrality analyses and neutral allele frequency test (Fu's $F_{s}$ ) of the Cyt $b$ and EF-1a gene regions in Phlebotomus and Sergentomyia species

\begin{tabular}{|c|c|c|c|c|c|}
\hline Species & P. papatasi & P. alexandri & Subgenus Sergentomyia & Subgenus Sintonius & \\
\hline Genes & Cyt b & Cyt b & Cyt b & $E F-1 a$ & Cyt b \\
\hline Fragment length (bp) & 788 & 789 & 789 & 497 & 789 \\
\hline$m$ & 6 & 5 & 7 & 6 & 6 \\
\hline$S$ & 431 & 428 & 367 & 276 & 35 \\
\hline$p_{\mathrm{s}}$ & 0.555 & 0.560 & 0.682 & 0.584 & 0.0445 \\
\hline$\Theta$ & 0.243 & 0.269 & 0.278 & 0.256 & 0.0296 \\
\hline$\pi$ & 0.332 & 0.335 & 0.375 & 0.305 & 0.0296 \\
\hline D & 2.391 & 1.876 & 2.041 & 1.250 & 0 \\
\hline A-T ratio (\%) & 70.92 & 73.84 & 68.4 & 46.82 & 70.02 \\
\hline G-C ratio (\%) & 29.08 & 26.16 & 31.6 & 53.18 & 29.98 \\
\hline G & 3.632 & 7.265 & 1.11 & 2 & 0.05 \\
\hline$K_{1}$ & 13.762 & 2.88 & 54.065 & 12.325 & 13.039 \\
\hline$K_{2}$ & 9.879 & 3.198 & 60.416 & 1.391 & 20.427 \\
\hline \multirow[t]{2}{*}{ Fu's $F_{\mathrm{s}}$} & 3.047 & 3.568 & Sergentomyia genus & & -0.463 \\
\hline & & & 0.913 & 1.482 & \\
\hline
\end{tabular}




\begin{tabular}{|c|c|c|c|c|c|c|c|c|c|c|c|c|c|c|c|c|c|c|c|c|c|c|c|c|c|c|}
\hline \multicolumn{10}{|c|}{} \\
\hline
\end{tabular}

Fig. 3 Pairwise comparisons of the nucleotide sequences among the species of Phlebotomus and Sergentomyia for the Cyt $b$ gene region based on the percent divergence (below diagonal) and identity (above diagonal) in the studied $\mathrm{CL}$ foci

between geographic distances and genetic variation based on the Mantel test $\left(R^{2}=0.3716, P=0.10\right)$. Therefore, the results show that geographic distance did not have a significant effect on the genetic differentiation observed in the Sergentomyia and Phlebotomus populations.

\section{Discussion}

Accurate identification of sand fly species based on molecular analyses is a valuable approach for determining the epidemiological aspects and distribution of natural populations in unexamined areas. In our investigation, using phylogenetic analysis, molecular characterization was performed to identify the population structure of the sand flies that were collected along the Khuzestan borders, which distinctly revealed the previously unrecorded species $P$. sergenti to be in a separate clade of the $C y t b$ region (Fig. 4). This study shows that each sand fly species includes unique sequences of the nuclear $(E F-$ $1 \alpha)$ and mitochondrial $(C y t b)$ gene markers. Certain sand fly species, such as $P$. papatasi and $P$. alexandri, featured a greater number of different haplotypes than the other sand fly species, which is related to specific geographical distances (Dashte-Azadegan, Khorramshahr and Abadan).

Finding the non-native species $P$. sergenti in new areas where no record exists implies expanding distribution of the vectors and their adaptation to local habitat conditions or environmental modifications along the Khuzestan borders (Table 1). In addition, L. tropica had been isolated from human ulcers at the same locations in the
Dashte-Azadegan district (Susangerd and Howeyzeh) [31]. The two endemic districts of ZCL in Khuzestan Province, Khorramshahr and Shush, were studied investigating the causes of sand fly biodiversity [32] and $S$. christophersi was found as a new record. However, we could not locate $S$. christophersi from the western borders of Khuzestan Province or west of Shush city (Fig. 2), maybe Jahanifard et al. [32] found S. christophersi in the east, not in the west of Shush area. Moreover, the GenBank database does not contain a records for S. dentata, S. iranica, S. baghdadis and S. tiberiadis and a verified record of $S$. antennata sequences for the $C y t b$ gene marker.

The transition/transversion (ts/tv) rate ratios for $P$. papatasi are $k 1=13.726$ for purines and $k 2=9.879$ for pyrimidines (Table 4). Further, the overall transition/ transversion bias is $R=4.866$ within $P$. papatasi, and the overall $(\mathrm{ts} / \mathrm{tv})$ rate is lower in $P$. alexandri sequences $(R$ $=1.18$ ) for the $C y t b$ gene, perhaps due to postmutation processes from the high mutation rate of methylated cytosines to thymine in the P. papatasi population. However, heterogeneity manifestations exhibit a selective advantage for survival and fitness in the sand fly population sequences along the Khuzestan borders (Table 4).

The number of interpretable sequences for the $E F$ $1 \alpha$ gene in P. papatasi, $P$. alexandri and the subgenus Sintonius was less than three, and the quality of the remainder was insufficient to evaluate the DNA fragments after resequencing. Perhaps this phenomenon is due to the presence of multiple loci or two copies in these species as a result of gene duplication events 


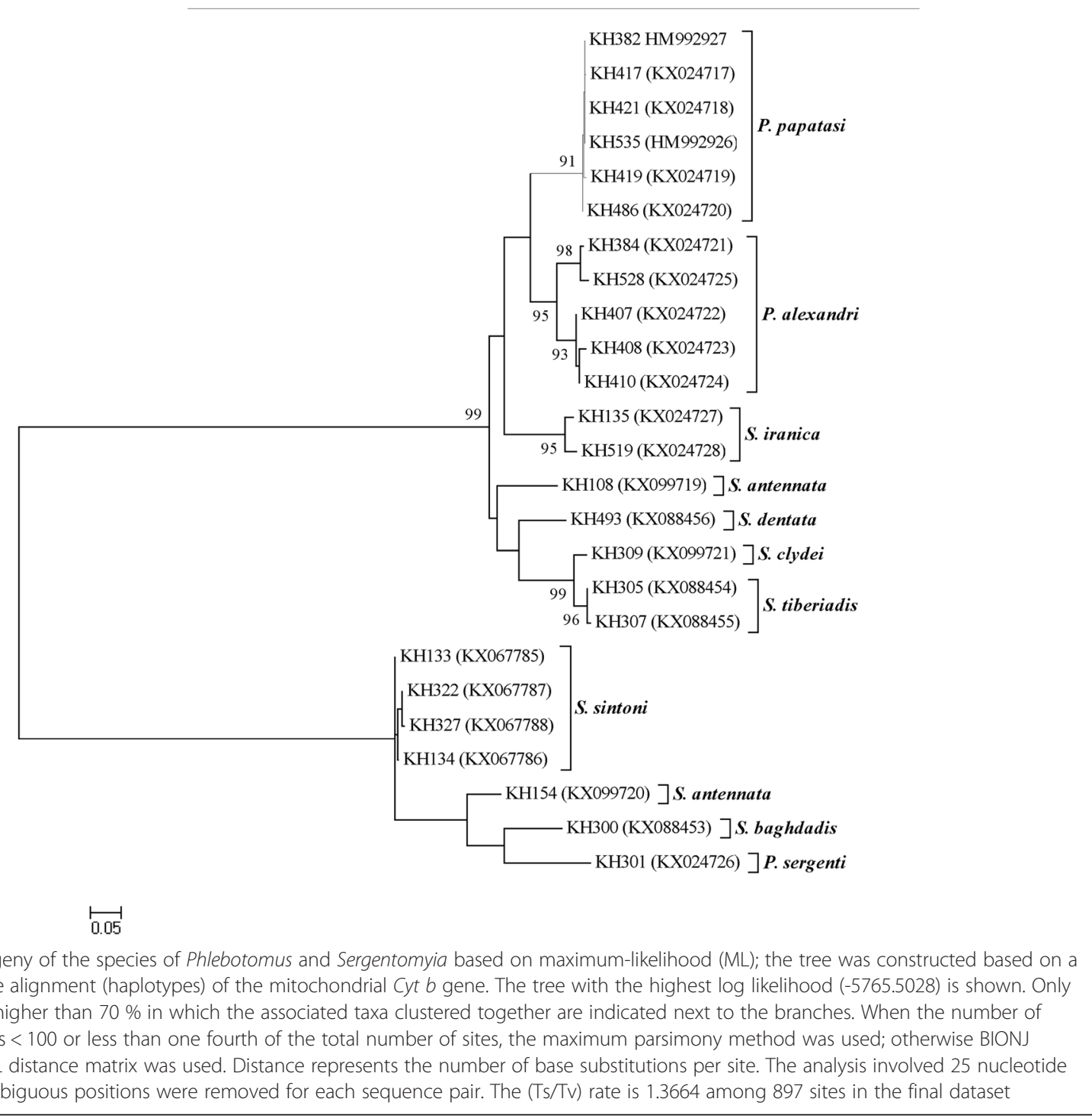

[10]. Therefore, the sequences were excluded from statistical analyses. The estimated overall (ts/tv) bias for the subgenus Sergentomyia is $R=3.31$. Tajima's $D$ value of the nuclear gene $(E F-1 \alpha)$ indicated low levels of polymorphisms $(D>+1, P=1.250596)$ compared with the mitochondrial $C y t \quad b$ gene $(D>+2, \quad P=$ 2.041410) (Table 4). This phenomenon may be due to the nature of the nuclear gene $(E F-1 \alpha)$ and the low number of nucleotide substitutions between the species of a single subgenus, which are homogenized by gene conversion [15]. The $E F-1 \alpha$ gene is multi copy number, this may be there are significant differences between the studied individuals and not an actual difference.

Although the data of Tajima's $D$ test for the $C y t b$ gene of $P$. papatasi $(D>+2)$ revealed higher nucleotide diversity than that observed for $P$. alexandri and the genus
Sergentomyia (more mutations or high polymorphisms between pairs than the number of segregating sites), different haplotypes of $P$. papatasi and $P$. alexandri were positioned in their own clades with similar rates of haplotype diversity (HD: 0.933 and 1 for $P$. papatasi and $P$. alexandri, respectively) (Fig. 4). In spite of different values of Tajima's $D$, the haplotype diversity was nearly the same for all species for both $C y t b$ and $E F-1 \alpha$ genes $(\mathrm{HD}=1)$. The positive Tajima's $D$ value in Khorramshahr and Abadan could be due to a relative population decrease, subdivision or recent bottlenecking among $P$. papatasi, $P$. alexandri and Sergentomyia spp. (Table 4). Positive values of Fu's $F_{\mathrm{s}}$ demonstrated allele deficiency because of overdominant selection or recent population bottleneck for $P$. papatasi (Fu's $F_{s}=3.047$ ), $P$. alexandri (Fu's $F_{s}=3.568$ ) and Sergentomyia spp. (Fu's $F_{s}=0.913$ ) [33]. Based on the interpretation of tree topology in the bottleneck stage, 


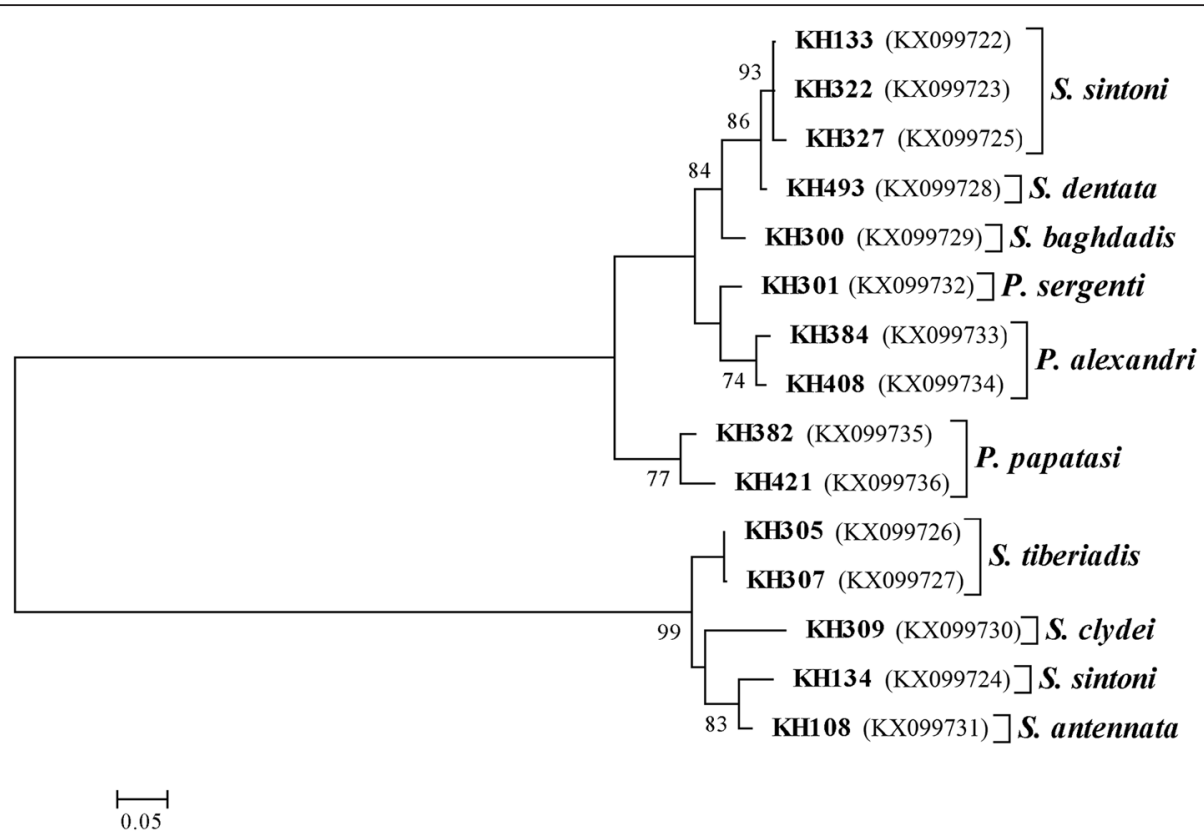

Fig. 5 The phylogeny of the species of Phlebotomus and Sergentomyia based on maximum-likelihood (ML); the tree was constructed based on a multiple sequence alignment (haplotypes) of the nuclear EF-1a gene. Only bootstrap values higher than $70 \%$ are indicated for each branch. Distance represents the number of base substitutions per site. The (Ts/Tv) rate is 1.3222 among 516 sites in the final dataset

several lineages have remained survivable under this stage without coalescing and consequently provide trees with long internal branches [33]. In fact, the topology of our phylogenetic tree obtained from the sand flies of Khuzestan borders (Figs. 4, 5) revealed the same pattern associated with the weak bottleneck stage and no effect on the fitness and survival of the sand flies. Additionally, the neutrality indices were significant for the genera Phlebotomus and Sergentomyia and this may result from selection pressure (the Z-test was $P<0.05$ and $\mathrm{dN} / \mathrm{dS}$ ratios were biased toward synonymous mutations among closely related species of sand flies) induced by environmental conditions. Pursuant to our molecular analyses, a selective sweep/hitch-hiking may have occurred in the subgenus Sintonius (S. clydei and S. tiberiadis) due to a population expansion or older bottlenecking, the negative values of Fu's $F_{\mathrm{s}}$ genotype (Fu's $F_{\mathrm{s}}$ $=-0.463$ ) indicating excess of low frequency haplotypes/alleles and beneficial mutation. We deduced that a balancing selection occurred due to a habitat modification (Iraq-Iran war). However, Tajima's $D$ value of the $C y t b$ gene regions indicates an equivalent number of pairwise nucleotide differences and segregating sites $(D=0)$ for the subgenus Sintonius, which indicates that the population was evolving as a per mutation-drift equilibrium and therefore, increasing the fitness of the Sintonius spp. The observed demographic changes between the populations of Sintonius spp. and other sand fly species can arise from random fluctuation of neutral mutation which likely goes up or down through genetic drift and demographic events. A decrease in genetic and phenotypic variation or conservation biology (loss of biological diversity) is possible for sand fly species due to human impact [34]. Human encroachment such as habitat fragmentation, burning, logging, the presence of domestic animals, using fertilizer, natural devastation, infrastructure deterioration and civil engineering due to developments of urbanization after the Iraq-Iran war can insert large scale exchange of individuals between populations. These interventions serve as an ever increasing threat to biodiversity.

Consistent with our molecular results, we observed the rapid expansion of nucleotide differences among sequences for Phlebotomus spp. and the remainder of the genus Sergentomyia (except Sintonius), with considerable random mutations and low genetic variation at a geographical location along the Khuzestan borders (23 haplotypes for the $C y t b$ gene and 15 haplotypes for EF-1 $\alpha$ from 108 individuals). Perhaps both, mitochondrial and nuclear genes, are involved in perfect harmony with balancing selection under imposed pressures from nature and human intervention. A GC-biased gene conversion may have occurred due to the presence of GC-rich isochores regions in the nuclear $E F-1 \alpha$ gene in comparison with the mitochondrial $C y t b$ gene. However, the observed corresponding selection could be expected for housekeeping genes, as a fundamental rule underlying molecular mechanisms in establishing proper conditions for life.

Considering landscape modifications in the attacked areas after military operations of war, increased genetic homogeneity or accumulation of similar set of alleles was expected. The high rate of allele frequency and 
polymorphisms among the sand fly sequences found herein may be considered as an effect of genetic drift associated with habitat fragmentation and a reduction in genetic variation (extrinsic selection).

The warfare between Iraq and Iran has ruined the natural habitat of phlebotomine sand flies as anthropogenic factors $[35,36]$ and has introduced non-native sand flies to the Khuzestan borders. Notably, genetic tractability and later adaptation to the current ecological and environmental features have rendered conditions more propitious for vector survival. Additionally, constant change in habitat quality and differences in the flying capacities of sand fly species are the crucial factor that affects sand fly dispersal, genetic structure and population responses along the Khuzestan boundaries, where the natural habitat was lost during war.

\section{Phylogenetic inference based on molecular analyses}

Phylogenetic analysis and molecular identification of the $E F-1 \alpha$ gene revealed less genetic structuring between the $P$. papatasi, $P$. alexandri and Sergentomyia populations than for the $C y t b$ gene across the Khuzestan borders. In contrast, an empirical examination of the $C y t b$ gene indicated higher genetic variation in the populations of $P$. papatasi, $P$. alexandri and a number of species of the genus Sergentomyia. All closely related sand fly species, except certain species of the subgenus Sergentomyia, produced a higher bootstrap likelihood (> $70 \%$ ) of developing reproductive isolation (Figs. 4, 5). The constructed tree for the nuclear $E F-1 \alpha$ gene produced the same results as the subgenus Sergentomyia for the Cyt $b$ gene but showed a better clade for Paraphlebotomus spp. than $C y t b$; based there on, $P$. sergenti and $P$. alexandri were grouped in a monophyletic clade (Fig. 4). Further, the specimens of KH421 and KH382, identified as unique and common haplotypes of $C y t b$ were placed in a well-supported branch of the phylogenetic tree for the nuclear $E F-1 \alpha$ genotype as well. The apparent discordance in nucleotide diversity between the $C y t b$ and $E F-1 \alpha$ sequences portends high variation in the maternally inherited mitochondrial gene due to elevated mutation [37]. A comparative study between the mtDNA $(C y t b)$ and nuclear gene $(E F-1 \alpha)$ was performed using the phylogeny of Larrossius spp., but no consistent data were generated [11]. The distribution of various sand fly species, particularly $P$. papatasi and $P$. alexandri, from one location patch to another, denotes not only for physical eligibility, but also for population dynamics $[38,39]$ due to re-establishment of sand fly populations or wildlife corridor as a possible mitigation instead of an ever-increasing threat to biodiversity of habitat fragmentation after the 8-year Iraq-Iran war.

\section{Conclusion}

We drew more attention to the effect of the combination of human activities and new environmental effects at the Iran-Iraq borders on vector genotypes as well as the selection and emergence of existing genotypes. The neutrality and molecular analyses suggest that our findings provide compelling evidence of randomly evolving DNA sequences from both mitochondrial and nuclear DNA haplotypes to support the fitness and survival of wild-caught sand flies with neutral mutation. Further investigation of different sand fly species of the old and new world is necessary to determine the evolution, behavior and vector competence of unknown or nonnative sand fly species due to various environmental conditions and in different regions around the world, which have not yet been studied.

\section{Abbreviations \\ CIM, clustering integration method; CL, cutaneous leishmaniasis; Cyt b, cytochrome b; EF-1a, elongation factor 1alpha; HD, haplotype diversity; mtDNA, mitochondrial DNA; PCA, principal component analysis; ts/tv, transition/transversion; ZCL, Zoonotic Cutaneous Leishmaniasis.}

\section{Acknowledgements}

The sand fly collections were made possible by the assistance of the Centre of Health Services of Shush, Dasht-e Azadegan, Ahvaz, Shadegan, Abadan and Khorramshahr. We extend our thanks to Somayeh Mohammadi and Mehdi Baghban for helping with the laboratory and the field work.

\section{Funding}

This work was funded by the Pasteur Institute of Iran (grant no. 605) and was awarded to Prof. Parviz Parvizi.

\section{Availability of data and material}

Essential data are presented in the main text of the paper. Nucleotide sequence data reported in this paper are available in the GenBank, EMBL, and DDBJ databases under accession numbers KX024717-KX024728; KX067785-KX067788 and KX088453-KX088456 (Cyt b gene) and KX099722-KX099736 (EF-1a gene).

\section{Authors' contributions}

$P P, S E$ and $A B$ conceived and designed the experiments. $S E$ and $A B$ performed the fieldwork. SE identified the sand fly specimens

morphologically and performed the molecular laboratory experiments. PP, SE and $A B$ analyzed the data and prepared the manuscript. All authors read and approved the final version of the manuscript.

\section{Competing interests}

The authors declare that they have no competing interests.

Ethics statement and consent for publication

Not applicable.

Received: 29 February 2016 Accepted: 23 May 2016

Published online: 03 June 2016

\section{References}

1. Ready PD. Biology of phlebotomine sand flies as vectors of disease agents. Annu Rev Entomol. 2013;58:227-50.

2. Rogers ME, Bates PA. Leishmania manipulation of sand fly feeding behaviour results in enhanced transmission. PLoS Pathog. 2007;3, e91.

3. Ready PD, Rogers ME: Behaviour of sand flies infected with Leishmania. In: Ecology and control of vector-borne diseases. 2012, Vol. 3: Ecology of vector-parasite interactions, ed. CJM Koenraadt, W Takken. Wageningen, The Neth.: Wageningen Academic Publishers.

4. Maroli M, Feliciangeli MD, Bichaud L, Charrel R, Gradoni L. Phlebotomine sand flies and the spreading of leishmaniases and other diseases of public health concern. Med Vet Entomol. 2013;27:123-47.

5. Nadim N, Javadian A, Mohebali M, Momeni AZ. Leishmania and leishmaniasis. 3rd ed. Iran: Iran University Press; 2008. 
6. Hamarsheh O. Distribution of Leishmania major zymodemes in relation to populations of Phlebotomus papatasi sand flies. Parasit Vectors. 2011;4:1-6.

7. Alvar J, Vélez ID, Bern C, Herrero M, Desjeux P, Jannin J, den Boer M. Leishmaniasis worldwide and global estimates of its incidence. PLoS One. 2012;7:e35671

8. Shirzadi M: Instruction of cutaneous leishmaniasis care (Salak) in Iran. M.O.H M.E, Deputy of Health, Administration Center of Contagious Diseases, Office of Transmissible Disease between Humans and Animals. First Pub, Raze nahan 2012, Tehran. 114 p.

9. Mahamdallie SS, Pesson B, Ready PD. Multiple genetic divergences and population expansions of a Mediterranean sand fly, Phlebotomus ariasi, in Europe during the Pleistocene glacial cycles. Heredity. 2011;106:714-26.

10. Parvizi $P$, Assmar M. Nuclear elongation factor 1-a gene, A molecular marker for Iranian sand fly identification. Iran J Public Health. 2007;36:25-37.

11. Esseghir S, Ready PD, Ben-Ismail R. Speciation of Phlebotomus sand flies of the subgenus Larroussius coincided with the late Miocene-Pliocene aridification of the Mediterranean subregion. Biol Jo Linn Soc 2000;70:189-219.

12. Parvizi P, Tahehrkhani H, Ready PD. Phlebotomus caucasicus and Phlebotomus mongolensis (Diptera: Psychodidae): indistinguishable by the mitochondrial cytochrome b gene in Iran. B Entomol Res. 2010;100:415-20.

13. Testa JM, Montoya-Lerma J, Cadena H, Oviedo M, Ready PD. Molecular identification of vectors of Leishmania in Colombia: mitochondrial introgression in the Lutzomyia townsendi series. Acta Trop. 2002;84:205-18.

14. Parvizi P, Ready PD. Molecular investigation of the population differentiation of Phlebotomus papatasi, important vector of Leishmania major in different habitats and regions of Iran. Iran Biomed J. 2006;10:69-77.

15. Clouse RM, Sharma PP, Giribet G, Wheeler WC. Elongation factor-1 a, a putative single-copy nuclear gene, has divergent sets of paralogs in an arachnid. Mol Phylogenet Evol. 2013;68:471-81.

16. Monteiro A, Pierce NE: Phylogeny of Bicyclus (Lepidoptera: Nymphalidae) inferred from COI, COII, and EF-1a gene sequences. Mol Phylogenet Evol doi:10.1006/mpev.2000.0872

17. Hines HM, Cameron SA, Williams PH. Molecular phylogeny of the bumblebee subgenus Pyrobombus (Hymenoptera: Apidae: Bombus) with insights intogene utility for lower-level analysis. Invertebr Syst. 2006;20:289-303

18. Regier JC, Shultz JW, Zwick A, Hussey A, Ball B, Wetzer R, Cunningham CW. Arthropod relationships revealed by phylogenomic analysis of nuclear protein-coding sequences. Nature. 2010;463:1079-83.

19. Nzelu CO, Kato H, Puplampu N, Desewu K, Odoom S, Wilson MD, et al. First detection of Leishmania tropica DNA and Trypanosoma species in Sergentomyia sand flies (Diptera: Psychodidae) from an outbreak area of cutaneous leishmaniasis in Ghana. PLoS Neglect Trop D. 2014;8, e2630.

20. Depaquit J. Molecular systematics applied to phlebotomine sand flies: review and perspectives. Infec Genet Evol. 2015.

21. Mahamdallie SS, Ready PD. No recent adaptive selection on the apyrase of Mediterranean Phlebotomus: implications for using salivary peptides to vaccinate against canine leishmaniasis. Evol Appl. 2012:5:293-305.

22. Ebrahimi S, Bordbar A, Esmaeili Rastaghi AR, Parvizi P. Spatial distribution of sand fly species (Psychodidae: Phlebotominae), ecological niche, and climatic regionalization in zoonotic foci of cutaneous leishmaniasis, southwest of Iran. J Vector Ecol. 2016:41(1):xxx [Accepted galley proof].

23. Alexander B. Sampling methods for phlebotomine sand flies. Med Vet Entomol. 2000:14:109-22.

24. Parvizi P, Benlarbi M, Ready PD. Mitochondrial and Wolbachia markers for the sand fly Phlebotomus papatasi: little population differentiation between peridomestic sites and gerbil burrows in Isfahan province, Iran. Med Vet Entomol. 2003;17:351-62.

25. Parvizi P, Mauricio I, Aransay AM, Miles MA, Ready PD. First detection of Leishmania major in peridomestic Iranian sand flies: comparison of nested PCR of nuclear ITS ribosomal DNA and semi-nested PCR of minicircle kinetoplast DNA. Acta Trop. 2005;93:75-83.

26. Ready PD, Lainson R, Shaw JJ, Souza AA. DNA probes for distinguishing Psychodopygus wellcomei from Psychodopygus complexus (Diptera: Psychodidae). Mem I Oswaldo Cruz. 1991;86:41-9.

27. Kumar S, Gadagkar SR. Disparity Index: A simple statistic to measure and test the homogeneity of substitution patterns between molecular sequences. Genetics. 2001;158:1321-7.

28. Tamura K, Peterson D, Peterson N, Stecher G, Nei M, Kumar S. MEGA5: Molecular evolutionary genetics analysis using maximum likelihood, evolutionary distance, and maximum parsimony methods. Mol Biol Evol. 2011:28:2731-9.

29. Guindon S, Gascuel O. A simple, fast and accurate algorithm to estimate large phylogenies by maximum likelihood. Syst Biol. 2003;52:696-704

30. Libardo P, Rozas J. DnaSP v5: software for comprehensive analysis of DNA polymorphism data. Bioinformatics. 2009;25:1451-2.

31. Spotin A, Rouhani S, Parvizi P. The associations of Leishmania major and Leishmania tropica aspects by focusing their morphological and molecular features on clinical appearances in Khuzestan province, Iran. Biomed Res Int 2014, ID 913510, 13 pages doi:10.1155/2014/913510.

32. Jahanifard E, Yaghoobi-Ershadi MR, Akhavan AA, Akbarzadeh K, Hanafi-Bojd AA, Rassi Y, et al. Diversity of sand flies (Diptera, Psychodidae) in southwest Iran with emphasis on synanthropy of Phlebotomus papatasi and Phlebotomus alexandri. Acta Trop. 2014;140:173-80.

33. Ramı'rez-Soriano A, Ramos-Onsins SE, Rozas J, Calafell F, Navarro A. Statistical power analysis of neutrality test under demographic expansions, contractions and bottlenecks with recombination. Genetics. 2008;179:555-67.

34. Soulé ME. Thresholds for survival: Maintaining fitness and evolutionary potential. In: Conservation Biology: An Evolutionary-Ecological Perspective. Sunderland, MA, Sinauer. 1980; 111: 124.

35. Valderrama A, Tavares MG, Andrade Filho DJ. Anthropogenic influence on the distribution, abundance and diversity of sand fly species (Diptera: Phlebotominae: Psychodidae), vectors of cutaneous leishmaniasis in Panama. Mem I Oswaldo Cruz. 2011;106:1024-31.

36. Galtier N, Nabholz B, Glémin S, Hurst GD. Mitochondrial DNA as a marker of molecular diversity: a reappraisal. Mol Ecol. 2014:18:4541-50.

37. Simon C, Frati F, Beckenbach A, Crespi B, Liu H, Flook P. Evolution, weighting and phylogenetic utility of mitochondrial gene sequences and a compilation of conserved polymerase chain reaction primers. Ann Entomol Soc Am. 1994;87:651-701.

38. Dunning JBJ, Stewart DJ, Danielson BJ, Noon BR, Root TL, Lamberson RH, Stevens EE. Spatially explicit population models: current forms and future uses. Ecol Appl. 1995;5:3-11.

39. Hanski I. Metapopulation ecology. Oxford: Oxford University Press; 1999. ISBN 0-19-854065-5.

\section{Submit your next manuscript to BioMed Central and we will help you at every step:}

- We accept pre-submission inquiries

- Our selector tool helps you to find the most relevant journal

- We provide round the clock customer support

- Convenient online submission

- Thorough peer review

- Inclusion in PubMed and all major indexing services

- Maximum visibility for your research

Submit your manuscript at www.biomedcentral.com/submit
C Biomed Central 